Chapter 13

\title{
Adjuvant Treatment in Colorectal Cancer
}

\author{
Krassimir Ivanov, Nikola Kolev, Ivan Shterev, \\ Anton Tonev, Valentin Ignatov, Vasil Bojkov and \\ Tanya Kirilova
}

Additional information is available at the end of the chapter

http://dx.doi.org/10.5772/56914

\section{Introduction}

Worldwide, more than 1 million people develop colorectal cancer (CRC) annually [1]. CRC is a major health problem in the Western world and the second most common cause of cancer mortality [2]. To improve performance, the role of chemotherapy for CRC has increased dramatically over the last decade. Of course surgery remains the cornerstone of treatment, the vast majority of CRC patients now receive chemotherapy with multiple agents that are currently approved for the treatment in the appropriate setting. However, it is a complex process to select the optimal chemotherapy for each patient and practice evidence gap is still a problem. We found large differences in patterns of institution, region and country. The results suggest that the lack of evidence for CRC chemotherapy practice still exists around the world. [3] Recently, standardization of cancer treatment, including chemotherapy, has become of particular importance for the quality of cancer therapy. It is important to know whether the overhaul performed normalization of CRC chemotherapy. Measures and quality indicators are needed and several studies on indicators of quality of cancer care have been reported. However, measures to assess the standardization of cancer therapy are not well established. In this study, we evaluated the usefulness of the oncology market research to assess the evidence gap in practice CRC chemotherapy. We also discuss the role of the method to measure the effect of normalization of CRC chemotherapy. $[4,5]$

Although surgery remains the cornerstone of treatment, the vast majority of CRC patients now receive chemotherapy to reduce the risk of metastatic spread by eradicating microscopic tumor foci that are distant from the primary tumor and undetectable in 
perioperative assessment of tumor extension. [5] Five-year survival of patients are mainly determined by the histological stage of the tumor at the time of resection. The most important prognostic factor for survival in patients without visceral metastases is the stage of the tumor determined by the depth of penetration of the tumor in the bowel wall and the number of lymph nodes [4] (lymph nodes $>12$ examined). Result of the meta - analysis of over 10 studies showed that each - two-month delay of adjuvant chemotherapy resulted in a $14 \%$ decrease in overall survival, suggesting that adjuvant chemotherapy should be administered as soon as possible [5].

The introduction of new cytotoxic agents such as oral fluoropyrimidines, oxaliplatin and irinotecan in chemotherapy (CT) regimens have improved the response rate, disease-free survival (DFS) and overall survival (OS) in patients with metastatic colorectal cancer. [6, 7] This has encouraged the trials in the adjuvant treatment of non-metastatic disease, especially in patients with stage III tumors. Table 1 shows the most common regimens used for the CT adjuvant. Surgery alone is usually curative for colon cancer stage II, but about $20 \%$ to $30 \%$ of these patients develop recurrence and die of metastatic disease. [8] This underpins the need for prognostic factors such as microsatellite instability (MSI), which are potentially predictive of tumor response to cytotoxic agents. [7] Prognostic factors are particularly useful in the context of stage II colorectal cancer, where the benefits of cytotoxic adjuvant therapy are more controversial than in stage III disease. The identification of accurate and validated predictive and prognostic markers help clinicians in choosing appropriate use of adjuvant chemotherapy in patients with stage II CRC.

MSI is a change in the length of microsatellite DNA due to the insertion or deletion of repeating units - from 1to 5 nucleotides caused by defects in mismatch repair genes or methylation of their promoters. [9] Tumors with MSI are more often proximal, poorly differentiated, mucinous, and show a significant lymphocytic infiltration. [9] Colon cancers with high-frequency MSI have clinical and pathological features that distinguish them from microsatellite stable tumors and MSI is a marker of favorable outcome and a predictor for the benefit decreased from fluorouracil - based adjuvant chemotherapy in patients with stage II or colon cancer stage III with microsatellite stable tumors or tumors with lowfrequency microsatellite instability. [10] Silence or mutation of mismatch repair (MMR) genes can lead to protein deficiency MMR and MSI 10. This is observed in patients with Lynch syndrome, and it is a rare cause of hereditary colon cancer 2 to $4 \%$ of the 11 cases. Somatic mutation is reported in 19\% of CRC [12], while silencing of MMR genes can be observed in up to $52 \%$ of sporadic colon cancers [13].

In sporadic CRC, three tumor phenotypes were defined: microsatellite stable (MSS), lowfrequency MSI (MSI-L) and high frequency MSI (MSI-H). It has been reported that MSI-H are more frequently found in stage II disease than in stage III disease 14. This may partly explain the benefit decreased from 5 - fluorouracil adjuvant chemotherapy (5FU) in patients with stage II. Tente identify patients who might benefit from adjuvant chemotherapy led to the development of multigene tests as several Oncotype DX etc. Unfortunately, there is no evidence that any of them can predict the potential benefit of adjuvant chemotherapy. [13] 


\section{Treatment guidelines for CRC}

Some guidelines for the treatment of CRC have been developed to promote the standardization of CRC treatment. Only two drugs, panitumumab and cetuximab first line were still causing discord. Bevacizumab or capecitabine combined with oxaliplatin is used for the treatment of advanced and recurrent CRC, while the FOLFOX regimen is used in patients with a high risk of recurrence in the adjuvant chemotherapy. In this major revision, current controversies are treated as clinical issues with many references.

Adjuvant 5-FU chemotherapy is the standard treatment used in patients with stage III (Dukes C) and high-risk stage II (Dukes B) tumors. Capecitabine and bolus 5FU regimes have proven efficacy and are associated with a low risk of severe toxicity. The addition of oxaliplatin to 5FU improves patient outcomes in the adjuvant setting. Mosaic of randomized trials in 2246 patients with stage 2 or 3 of the CRC to receive LV5FU2 or FOLFOX-4 chemotherapy. The operating system after 6 years of follow-up for all patients was $78.5 \%$ for FOLFOX-4 against 76\% for LV5FU2. Subgroup analysis showed stage-specific 6-year OS rate of $72.9 \%$ against $68.7 \%$ in patients with stage 3 of the CRC and $86.9 \%$ compared with $86.8 \%$ in patients with stage 2 CRC for FOLFOX-4 and LV5FU2, respectively. The NSABP $\mathrm{C}-07$ trial had a similar design, the addition of oxaliplatin to 5FU adjuvant chemotherapy, but used a different calendar 5FU and oxaliplatin doses delivered also less than in the MOSAIC study (nine against twelve). The first results showed a similar improvement in disease-free survival (DFS) than that observed in the MOSAIC trial. A recently presented the final results confirm improved DFS, but showed shorter survival time after recurrence in the oxaliplatin arm and an improvement in overall survival has not been seen. A significant interaction between age and survival of certain parameters were observed. Patients less than 70 years appeared to benefit from the addition of oxaliplatin while patients over 70 years, no benefit was observed consistently. The analysis of the ACCENT database, including 10, 449 patients less than 70 years and 2170 patients over 70 years from six randomized trials have demonstrated a significant interaction between age and treatment effect. No differences in results were noted between experimental (combination) chemotherapy and chemotherapy in patients with fluoropyrimidine control over 70 years. Adding oxaliplatin to $5 \mathrm{FU}$ increases the overall incidence of grade 3 toxicity and is associated with the occurrence of peripheral sensory neuropathy. Over $90 \%$ of patients experience temporary symptoms typically induced cold, with a minority of patients with persistent symptoms affecting activities of daily living (grade 2 and 3 toxicity). In the MOSAIC study, grade 3 peripheral sensory neuropathy was noted in $12.5 \%$ of patients treated with oxaliplatin for the treatment. After 48 months of follow-up, rates of toxicity were grade $11.9 \% 1, \%$ [22] 8 degree and $0.7 \%$ grade 3 , respectively. Similar data were presented for the NSABP C-07 study.

Decisions regarding the use of adjuvant chemotherapy combinations are more complex. The incidence of approximately $3 \%$ of significant long-term peripheral neuropathy and MS MT Seymour Braun could interfere with the activities of the daily life of the patient influences decision-making in relation to the small additional benefit accrued to receive the 
oxaliplatin. MOSAIC and NSABP the C-07 trials delivered a total dose of oxaliplatin different, but both studies noted similar improvements in DFS. International trials evaluating shorter periods of oxaliplatin-based chemotherapy in the adjuvant setting (12 versus 24 weeks of chemotherapy oxaliplatin/5FU ISRCTN 59757862) in order to assess the noninferiority of short periods of treatment, as well as consideration of the evaluation criteria of quality of life. The relative benefit of chemotherapy is also a key factor in the choice of treatment for patients in the adjuvant setting. Patients with stage 3 are a heterogeneous group and decisions based on age, the relative risk of recurrence (N1 N2 against disease), and the additional benefit that can be achieved by adding oxaliplatin need to be carefully considered. Given the new data in patients over 70 years, it seems likely that chemotherapy oxaliplatin are used less frequently in this group. Patients with disease stage 2 have an excellent prognosis with or without chemotherapy 5FU-based and high-risk patients functions are selected for processing. Both C-07 and MOSAIC are powered to assess the benefit of adding oxaliplatin to 5-FU in patients with stage 2 disease, but a trend towards improved DFS was noted. However, no OS benefit is probably very low in absolute terms $(<2 \%)$ and difficult to justify given the excellent overall performance $(>80 \%$ at 5 years of operation) and the risk of neurotoxicity. [23]

\section{3. $5 \mathrm{FU}$}

In 1990, Moertel and colleagues first reported the value of adjuvant chemotherapy in patients with stage III colon cancer (Dukes C, Tx N + M0). [15] This study showed an increase in overall survival and progression-free survival in patients receiving 5FU/ Levamisole - based chemotherapy for 1 year against levamisole alone or without chemotherapy. With a median follow - up of 6.5 years, patients treated with 5-FU / levamisole showed a $40 \%$ reduction in recidivism and an estimated reduction of $33 \%$ of overall mortality [16]. 5-FU is a pyrimidine analogue, which inhibits thymidylate synthase (TS) (involved in the de novo synthesis of thymidine) and is involved in incorporation into RNA and DNA with the inhibition of DNA synthesis and function [17]. With 5FU bolus injection maximum concentration is reached in the plasma and bone marrow 100-1000 times higher than with continuous infusion. More than $85 \%$ of the administered drug is inactivated by dihydropyrimidine dehydrogenase (DPD), expressed mainly in the liver. Some mutation in DPD in about $2 \%$ in the general population can lead to serious life-threatening toxicity [17]. Leucovorin (LV) or folinic acid enhance the antitumor activity of 5FU. Today, there is a lack of LV in the United States, despite the absence of specific data confirming this statement. The QUASAR study investigators demonstrated that patients treated with 175 $\mathrm{mg}$ of LV similar survival and 3 - year recurrence rate of LV $25 \mathrm{mg}$ when administered as a bolus 5-FU as adjuvant therapy for CRC 7. Similar results have been reported in the parameters in metastatic CRC patients - there was no difference in survival or response rate in patients receiving 5FU bolus high - dose or low-dose LV [18]. Therefore, when LV is not available without LV treatment is reasonable. Comparison of monthly bolus FU / LV 
regimen (5FU bolus followed by LV 15 minutes) with double - monthly infusion LV5FU2 (LV for 2 hours, followed by bolus 5-FU, followed by a continuous infusion of 5-FU over 2 days) in 905 patients with stage II (43\%) or III (57\%) colon cancer showed that the regime was less toxic LV5FU2, especially regarding hematological and gastrointestinal events ( $P$ $<0.001$ ) [19]. No significant difference in DFS and OS was observed in one of two regi mens median follow-up 6 years. Two other studies have shown that the two ways of 5FU bolus administration combined with LV with or without levamisole and a continuous infusion of 5-FU are equivalent. Saini and colleagues conducted a multicenter randomized trial comparing the efficacy and toxicity of 12 weeks of 5FU delivered by continuous intravenous infusion against the standard bolus 5-FU and LV for 6 months as adjuvant treatment in colorectal cancer. The two schemes are equivalent, but the plan 12 weeks was less toxic [20]. The analysis of data from several trials in which patients were randomly resection of the tumor or tumor resection followed by adjuvant 5-FU/LV showed that the benefit of adjuvant chemotherapy was observed in patients with stage III [21,22], which suggests that because of nodal status of these patients are at higher risk. Both studies showed that the addition of oxaliplatin to 5-FU/LV as adjuvant therapy in stage II and elderly patients showed no significant DFS or OS $[23,24]$ benefits even in patients with characteristics high risk - T4 tumors, intestinal obstruction, venous invasion, etc. This suggests that, despite the failure of our definition of high-risk patients with stage II, 5-FU/LV regimen may be preferable. QUASAR investigators reported their analysis of 3238 patients, the majority of them were in stage II colon cancer. Patients were randomized to receive 5-FU/LV or observation. The relative risk of death from all causes in the 5-FU/LV arm versus observation was $0.82(95 \% \mathrm{CI}, 0.70$ to $0.95, \mathrm{p}=0.008)$. The relative risk of recurrence was $0.78(95 \%$ CI, 0.67-0, 91, p = 0.001) [7]. The investigators did not separate patients into individuals high or low risk. Risk factors are obstruction or bowel perforation, elevated preoperative CEA, poorly differentiated tumors, removal of CDC patients and MSI-S. The impact of adjuvant chemotherapy and the potential benefit of this new could not be clearly demonstrated in this study of patients with stage II.

\section{Oral fluoropyrimidines}

Two oral prodrugs of 5FU - capecetabine and uracil / tegafur (UFT), has demonstrated efficacy in metastatic disease, which is comparable with bolus 5-FU/LV regimens [25, 26]. After oral administration, capecitabine is rapidly absorbed with plasma concentrations peaking after 1.5 hours [27]. Pharmacokinetics is largely dose - dependent. The pharmacology of capecitabine is not significantly influenced by gender, race, performance status, body surface area, albumin or hepatic dysfunction [28]. The half-life of capecitabine is between 0.49 and 0.89 hours, whereas the half-life of the metabolite (5-FU) extends from 0.67 to 1.15 hours [29]. Regarding renal excretion primarily (more than $70 \%$ of metabolites), capecitabine is against - in patients with severe renal impairment (creatinine clearance less than 30 $\mathrm{mL} / \mathrm{min})$. 


\begin{tabular}{|c|c|c|c|}
\hline Therapy & Mechanism of Action & Indications & Potential Common Toxicities \\
\hline 5- Fluorouracil (5FU) & $\begin{array}{l}\text { Blocks the enzyme } \\
\text { thymidylate synthase (TS), } \\
\text { which is essential for DNA } \\
\text { synthesis }\end{array}$ & $\begin{array}{l}\text { Multiple uses in } \\
\text { combination with other } \\
\text { agents, both in the } \\
\text { adjuvant (postop) and } \\
\text { palliative setting }\end{array}$ & $\begin{array}{l}\text { Gastrointestinal (nausea, } \\
\text { diarrhea) } \\
\text { Myelosuppression } \\
\text { Fatigue }\end{array}$ \\
\hline Capecitabine & $\begin{array}{l}\text { Blocks thymidylate synthase } \\
\text { (orally administered prodrug } \\
\text { converted to } 5 \mathrm{FU} \text { ) }\end{array}$ & $\begin{array}{l}\text { Multiple uses in } \\
\text { combination with other } \\
\text { agents, both in the } \\
\text { adjuvant (postop) and } \\
\text { metastatic setting }\end{array}$ & $\begin{array}{l}\text { Gastrointestinal (nausea, } \\
\text { diarrhea) } \\
\text { Myelosuppression } \\
\text { Fatigue } \\
\text { Palmar-plantar syndrome } \\
\text { (hand-foot syndrome) }\end{array}$ \\
\hline Oxaliplatin & $\begin{array}{l}\text { Inhibits DNA replication and } \\
\text { transcription by forming } \\
\text { inter- and intra-strand DNA } \\
\text { adducts/cross-links }\end{array}$ & $\begin{array}{l}\text { Used in combination with } \\
5 F U, \text { leucovorin (LV) } \\
\text { (FOLFOX) in the adjuvant } \\
\text { (postop) and metastatic } \\
\text { setting }\end{array}$ & $\begin{array}{l}\text { Peripheral neuropathy } \\
\text { Gastrointestinal (nausea, } \\
\text { diarrhea) } \\
\text { Fatigue } \\
\text { Myelosuppression } \\
\text { Hypersensitivity }\end{array}$ \\
\hline Irinotecan & $\begin{array}{l}\text { Inhibits topoisomerase I, an } \\
\text { enzyme that facilitates the } \\
\text { uncoiling and recoiling of } \\
\text { DNA during replication }\end{array}$ & $\begin{array}{l}\text { Used alone or in } \\
\text { combination with 5FU, LV } \\
\text { (FOLFIRI) in the metastatic } \\
\text { setting }\end{array}$ & $\begin{array}{l}\text { Cholinergic (acute diarrhea) } \\
\text { Gastrointestinal (nausea, } \\
\text { late diarrhea) } \\
\text { Fatigue } \\
\text { Myelosuppression } \\
\text { Alopecia }\end{array}$ \\
\hline Bevacizumab & $\begin{array}{l}\text { Monoclonal antibody which } \\
\text { binds to VEGF ligand }\end{array}$ & $\begin{array}{l}\text { Used in combination with } \\
\text { either FOLFOX or FOLFIRI in } \\
\text { the metastatic setting }\end{array}$ & $\begin{array}{l}\text { Hypertension } \\
\text { Arterial thrombotic events } \\
\text { Impaired wound healing } \\
\text { Gastrointestinal perforation }\end{array}$ \\
\hline Cetuximab & $\begin{array}{l}\text { Monoclonal antibody to } \\
\text { EGFR (chimeric) that blocks } \\
\text { the ligand-binding site }\end{array}$ & $\begin{array}{l}\text { Used with irinotecan or as a } \\
\text { single agent in the } \\
\text { metastatic setting }\end{array}$ & $\begin{array}{l}\text { Acneform rash } \\
\text { Hypersensitivity } \\
\text { Hypomagnesemia } \\
\text { Fatigue }\end{array}$ \\
\hline Panitumumab & $\begin{array}{l}\text { Monoclonal antibody to } \\
\text { EGFR (fully humanized) that } \\
\text { blocks the ligand-binding } \\
\text { site }\end{array}$ & $\begin{array}{l}\text { Used as a single agent in } \\
\text { the metastatic setting }\end{array}$ & $\begin{array}{l}\text { Acneform rash } \\
\text { Hypomagnesemia } \\
\text { Fatigue }\end{array}$ \\
\hline
\end{tabular}

Table 1. Types of therapeutical agents and their mechanism of action

The X-ACT (Xeloda (capecitabine) in adjuvant therapy of colon cancer) Phase III trial in patients $(\mathrm{N}=1.987)$ compared capecitabine $(2500 \mathrm{mg} / \mathrm{m} 2$ / day, 14 to 21 days $)$ for bolus 5-FU/ 
LV. After a median follow-up of 6.9 years, both treatments showed similar efficacy in terms of DFS and OS [30]. The HR for DFS of capecitabine compared 5-FU/LV was 0.88 (95\% CI, 0.77 to 1.01), the upper limit of the $95 \%$ is well below the predefined non-inferiority margin of 1.20 ( $p<0.0001$ ) [30]. The 5-year DFS rate for capecitabine and 5-FU/LV were $60.8 \%$ and $56.7 \%$, respectively. These monitoring data confirm that, as adjunctive therapy for patients with colon cancer resected stage III oral capecitabine is at least equivalent to iv bolus treatment of 5FU/LV in terms of 5 - year DFS, the primary endpoint of X-ACT study. Infusional 5-FU regimens are now often favored because they offer similar efficacy or improved slightly bolus 5-FU/LV regimes and are generally better tolerated [31]. However, the plasma concentration profile of capecitabine is administered twice daily for 14 days, closer to that of a continuous infusion of 5-FU bolus injections as daily or weekly 5FU.La capecitabine Profile improved safety compared to bolus 5-FU/LV in terms of significantly lower rates of diarrhea, stomatitis, neutropenia, nausea, alopecia, febrile neutropenia [30].

UFT and oral LV was evaluated in the adjuvant setting in the NSABP C-06 trial. More than 1000 patients with colon cancer were randomized to receive either oral or intravenous UFT with LV 5FU with LV. $47 \%$ of patients had colon cancer stage II, and $53 \%$ had colon cancer stage III. Median follow-up was 62.3 months time. There was no significant difference in disease-free survival - or overall showing that the UFT is an acceptable alternative to parenteral 5-FU/LV [32]. No difference in toxicity profiles of the two regimens has been reported.

\section{Adjuvant therapy combination}

The hypothesis that the antitumor activity of the combination agent, including oxaliplatin, irinotecan, bevacizumab, cetuximab in metastatic cure rates would result in increased adjuvant proved to be often wrong. Oxaliplatin is a platinum compound and third generation of the safe administration of evidence of clinical activity has been reported 33 . Platinum compounds exert their effect through the development of covalent adducts with cellular DNA, which is not portable - specific cycle [34]. Platinum derivative oxaliplatin is described as having a "tri-exponential" reason for removing the half - life being successively 0.28 hours, 16.3 hours and 273 hours [35]. The fact that the third half-life of oxaliplatin hundreds of hours, the accumulation of the drug in the tissues can reasonably be expected. In this regard, one study examined the long - term retention of platinum 8-75 months after treatment with cisplatin and oxaliplatin [36]. Narrow therapeutic index of oxaliplatin and adverse reactions are mainly reported in the hematopoietic system, peripheral nerves, and gastrointestinal tract [35]. The addition of oxaliplatin to 5FU improves patient outcomes in the adjuvant setting. Mosaic of randomized 2246 patients with stage II or III CRC will LV5FU2 or FOLFOX-4 (which is LV5FU2 chemotherapy plus oxaliplatin on day 1]. The 5-year DFS rate of Phase II and III patients were $73.3 \%$ and $67.4 \%$ in the FOLFOX-4 groups and LV5FU2, respectively (RR 0.80, 95\% CI, 0.68-0, 93, p = 0.003) [37]. Subgroup analysis showed stage - specific 6 years OS rate of $72.9 \%$ against $68.7 \%$ in patients with stage III CRC (HR 0.80, 95\% CI, 0.65-0, 97, $\mathrm{P}=0.023$ ) and $85.0 \%$ against $83.3 \%$ in patients with stage II CRC $(\mathrm{P}=0.65$ ] in the FOLFOX-4 and LV5FU2, respectively [38]. As 
expected, the toxicity of the regimen FOLFOX-4 was higher than that observed in the LV5FU2 arm. All - cause mortality in the first 60 days was the same in both arms.The NSABP C-07 trial had a similar design, the addition of oxaliplatin to 5-FU adjuvant chemotherapy, but using a different calendar 5FU and also provide fewer doses of oxaliplatin in the MOSAIC study (nine against twelve). FLOX regimen in this trial was studied - oxaliplatin was given on weeks 1, 3, and 5 more per week 5-FU/LV bolus of 1-6 weeks, repeated at 8 week cycle, depending on the standard weekly 5-FU/LV treatment. Over 2000 patients were randomized to receive 5-FU/LV and FLOX treatment. Stage II patients were $29 \%$ and stage III patients was $71 \%$. The median duration of follow up was 34 months. The hazard ratio of FLOX against 5-FU/LV was 0.79 (95\% CI, 0.67 to 0.93], with a risk reduction of $21 \%$ in favor of FLOX [39]. As expected, treatment toxicity FLOX was higher than that observed in the 5-FU/LV arm. 15 deaths were recorded in the treatment with FLOX and 14 deaths 5-FU/LV. Update this study showed that the benefit of FLOX in DFS was observed in 7 - year median follow - but there was no significant difference in overall survival when the two arms were compared 24 (HR, $0.88,95 \% \mathrm{CI}, 0.74$ to $1.05, \mathrm{P}=$ 0.1428). A significant interaction between age and survival of certain parameters were observed. Patients less than 70 years appeared to benefit from the addition of oxaliplatin while in patients over 70 years, no benefit was observed consistently. MOSAIC and NSABP the C-07 trials delivered a total dose of oxaliplatin different, but both studies noted similar improvements in DFS. International trials evaluating shorter periods of oxaliplatin - based chemotherapy in the adjuvant setting (12 versus 24 weeks of chemotherapy oxaliplatin/5FU ISRCTN 59757862) in order to assess the non-inferiority of short periods treatment.Both NSABP C-07 and MOSAIC are powered to assess the benefit of adding oxaliplatin to 5FU in patients with stage II disease, but a trend towards improved DFS was noted.However, no OS benefit is probably very low in absolute terms $(<2 \%)$ and difficult to justify given the excellent overall performance ( $>80 \%$ at 5 years of operation) and the risk of neurotoxicity. Analysis of a phase III trial comparing capecitabine plus oxaliplatin (XELOX) with bolus 5-FU/LV as adjuvant treatment for colon cancer stage III showed that XELOX was an improvement of 3 years compared to 5-FU/LV DFS rate [40, 41]. Patients receiving XELOX had less adverse reactions such as diarrhea, alopecia, and more neurosensory toxicity, vomiting and hand-foot syndrome than patients receiving FU / LV. All these studies suggest that FOLFOX, XELOX FLOX and can be used interchangeably in contexts adjuvant. Irinotecan is a semisynthetic analogue of camptothecin, originally isolated from the China / Tibet ornamental tree Camptotheca acuminata.It is a chemotherapy agent that causes destruction of cells in S phase-specific topoisomerase I poison in the cell [42]. CALGB 89803 trial by Saltz and colleagues randomized 1264 patients to receive standard weekly bolus 5FU / LV bolus regimen or weekly irinotecan and 5-FU bolus / LV. The primary endpoints of the study were overall survival and disease-free survival. Surprisingly, they found no difference in either DFS (0.84] or OS $(\mathrm{P}=0.74$ ] between the two treatment arms with lethal and non-lethal toxicity increased by the addition of irinotecan to standard 5FU / LV pattern 43.This trial showed the need for randomized controlled trials adjuvant because advances in the treatment of metastatic disease does not necessarily translate into advances in adjuvant therapy. Phase III trial was conducted by large investigators PETACC-3. They 
investigated whether the addition of irinotecan to LV5FU2 would improve disease-free survival in patients with colon cancer. After surgery, patients with stage II and III colon cancer were randomized to receive surgery LV5FU2 (LV $200 \mathrm{mg} / \mathrm{m} 2$ infused over 2 hours, followed by 5-FU as a $400 \mathrm{mg} / \mathrm{m} 2$ bolus and then one of $600 \mathrm{mg} / \mathrm{m} 2$ by continuous infusion over 22 hours on days 1 and 2 every 2 weeks for 12 cycles) with or without irinotecan (180 mg / m 2 infused over 30 to 90 minutes, day 1, every 2 weeks) [44]. After a median follow-up of 66.3 months, the rate at 5 years was $56.7 \%$ with DFS irinotecan/ LV5FU2 and $54.3 \%$ for LV5FU2 alone $(\mathrm{p}=0.106)$. They observed that the addition of irinotecan to LV5FU2 was associated with an increased incidence of adverse reactions and neutropenia. They concluded that irinotecan added to LV5FU2 as adjuvant therapy does not confer a statistically significant improvement in overall survival or DFS in patients with colon cancer stage III versus LV5FU2 alone. For the moment, there are no data supporting the use of irinotecan-containing regimens in adjuvant stage II and III patients. Analysis PETACC-3 trial could not confirm the expected benefit of adding irinotecan in MSI-H patients. Bevacizumab is a recombinant humanized monoclonal antibody directed against the vascular endothelial growth factor (VEGF) which is used to inhibit the function of VEGF in vascular endothelial cells and thereby inhibit tumor angiogenesis-dependent solid tumors for growth and metastasis [45]. Bevacizumab has demonstrated clinical activity to increase in the standard CT metastatic settings.This led to the consideration of this agent in the adjuvant chemotherapy in the NSABP C-08 trial. More than 2500 patients, most of whom had stage III were randomized to receive FOLFOX6 modified 6 months, alone or with bevacizumab [46]. In the bevacizumab arm, bevacizumab was administered for more than 6 months, for a total of 1 year of bevacizumab.The primary endpoint of the study was 3 years DFS. The relative risk of FOLFOX plus bevacizumab versus FOLFOX alone was 0.89 (95\% CI, 0.76 to $1.04, p=0.15$ ). This study did not demonstrate the benefits of the use of bevacizumab in the adjuvant treatment of stage II and III CRC and for this reason the use of bevacizumab cannot be recommended for use in the adjuvant treatment of patients with colon cancer. Cetuximab is a monoclonal antibody capable of inhibiting the degradation and transmembrane receptor EGFR epidermal growth factor 47.Inhibition of EGFR is of major importance because EGFR control many important activities of tumor cells, including tumor growth and neo - angiogenesis, inhibition of the apoptotic response to chemotherapy and radiotherapy. In a Phase III, randomized, Alberts and colleagues evaluated the potential benefit of cetuximab added to the sixth amended plan FOLFOX.Ils randomized over 2500 patients to receive 12 cycles of FOLFOX every two weeks with or without cetuximab. The mutational status of the KRAS gene was decided at the central level. The median follow-up of 28 months. Three-year disease-free survival for FOLFOX alone was $74.6 \%$ against $71.5 \%$ with the addition of cetuximab (HR, 1.21, 95\% CI, 0.98 to $1.49, \mathrm{P}=0$ 08 ) in patients with wild-type KRAS, and $67.1 \%$ against $65.0 \%$ (HR 1.12, 95\% CI, 0.86 to $1.46, \mathrm{p}=0.38$ ) in patients with mutated KRAS 48.The trial did not demonstrate any benefit when adding cetuximab to FOLFOX regimen. More patients with grade 3 or higher adverse events $(72.5 \%$ versus $52.3 \%$, odds ratio (OR) $2.4,95 \% \mathrm{CI}, 2.1$ to $2.8, \mathrm{p}<.001$ ) and failure to carry Good 12 cycles (33\% versus 23\%, OR 1.6, 95\% CI, 1.4 to $1.9, \mathrm{p}<0,001$ ) were significant- 
ly higher with cetuximab.Increased toxicity was observed in patients aged 70 years or older. Therefore, the role of cetuximab in the adjuvant treatment is insignificant for the moment.

\section{Chemotherapy in elderly}

Colon cancer usually occurs in the elderly with a median age at diagnosis $>70$ years in the USA. Given the increasing life expectancy, patients aged $>75$ years will be an important component of oncology practice in the future. Despite this fact, very few patients $>75$ years participate in clinical trials. There is disagreement in the administration of standard adjuvant therapy between young and elderly patients, despite a significant survival benefit for most patients [49]. The pooled analyzes of safety and efficacy of adjuvant chemotherapy in the elderly showed comparable rates of toxicity and similar survival benefits compared to younger patients [50].The majority of data for adjuvant therapy in elderly patients is not the incorporation of new therapeutic agents such as oxaliplatin. Subsequent population-based studies have suggested that older people (eg the elderly category $<75$ years versus $>75$ years [51] were less likely to have received adjuvant therapy, but experienced similar survival rates compared younger patients [52]. Finally, although the majority of recommendations to reduce colorectal cancer screening for persons aged $<75$ years, the study results indicate that [53] patients aged $>75$ years account for almost $20 \%$ of cases of colon cancer lymph nodes.In this large populationbased study, investigators found that age was associated with significantly lower rates of adjuvant chemotherapy administration, whereas the survival benefits of such treatment are comparable to those of younger patients with stage III in [53] colon cancer.Although chronological age alone should not be an exclusion criterion, more work is needed to establish an optimal strategy and effective way to understand who would benefit most from adjuvant therapy after surgical resection.

\section{Efectivness}

Patients with metastatic CRC being treated with chemotherapy are followed closely to monitor efficacy. There are standardized efficacy measures, such as the RECIST (response evaluation criteria in solid tumors) criteria, used as endpoints for large clinical trials. A partial response is defined as a $30 \%$ decrease in the longest dimension of each measurable tumor deposit, using unidimentional, or RECIST criteria [54-56]. A complete response is complete disappearance of all clinically detectable disease. The response rate $(\mathrm{RR})$ is the percentage of patients who meet either a partial or complete response. Measures used to determine the duration of treatment benefit include:

1. progression-free survival (PFS), which is the time from the start of treatment to the date the disease, worsens;

2. disease-free survival (DFS), which is the length of time patients are free of disease after completion of curative treatment; and 
3. overall survival (OS), which is the length of time patients are alive after diagnosis or initiation of treatment for metastatic disease.

\section{Postoperative management}

Postoperative, or "adjuvant" systemic therapy has become standard for stage III colon cancer. Adjuvant therapy should also be strongly considered in stage II patients. It is generally recommended for any medically fit patient with stage II cancer with unfavorable factors, including colonic perforation, poorly differentiated histology, colonic obstruction, lympho vascular invasion, or inadequately sampled lymph nodes [61]. The optimal choice of adjuvant chemotherapy has recently changed from a 6-month course of 5FU-based chemotherapy alone to a 6-month course of infusional 5FU plus LV and oxaliplatin (FOLFOX) based on a large trial of adjuvant systemic therapy for resected stage II or III colon cancer [62].This trial demonstrated an increase in disease-free survival at 3 years from 72.9 to $78.2 \%(p=0.002)$ with addition of oxaliplatin to FU/LV. Five-year disease-free survival remained significant (HR: 0.80; $p=$ $0.003)$ and at 6 years there was an overall survival benefit for stage III patients $(68.3 \%$ versus $72.9 \%$ ) [64].Toxicities were comparable between the two groups, with the exception that oxaliplatin is associated with a much higher rate of paresthesia: $12.4 \%$ versus $0.2 \%$ grade 3 (serious) toxicity. This neurotoxicity persisted at a grade 3 level in $1.1 \%$ of treated patients at one-year of follow-up.

Many advances have occurred recently in the treatment of metastatic CRC. Active agents, in addition to the original 5FU, that have been approved by the Food \& Drug Administration (FDA) for mCRC include irinotecan, capecitabine, oxaliplatin, bevacizumab, cetuximab, and panitumumab. The goals of systemic therapy of mCRC include palliation of symptoms, prolongation of life, and in selected cases of liver-only metastases, tumor regression to facilitate surgical resection of these metastases. The median survival of a patient with $\mathrm{mCRC}$ has improved during the last decade from less than 1 year, with only 5FU-based therapy, to $\sim 2$ years, with multiagent systemic therapy.

5FU, often modified by LV, has been clinically used for half a century as a standard agent for mCRC [64]. This was the only available agent until 1996, when irinotecan was approved. Over the last decade, chemotherapies such as oxaliplatin and capecitabine and targeted agents such as bevacizumab, cetuximab, and panitumumab have been approved. 5FU blocks the enzyme thymidylate synthase (TS), which is essential for DNA synthesis. Leucovorin (LV), also known as folinic acid, enhances the antineoplastic effects of 5FU. Both LV (FOL = folinic acid) and 5FU ( $F=$ fluorouracil) can be combined with irinotecan (IRI) or oxaliplatin (OX) with the treatment acronyms FOLFIRI or FOLFOX, respectively. These alternative treatments consist of administration of a bolus of 5FU, LV, and either oxaliplatin or irinotecan. The patient is then sent home with a 2-day infusion of low-dose 5FU, administered by a small, lightweight, portable pump, usually worn on a belt or shoulder strap, infused through a centrally placed catheter. The patient or health care provider can simply disconnect the catheter after the 2-day infusion. Capecitabine is an oral fluoropyrimidine with a similar mechanism of action and similar efficacy as 5FU. 
Irinotecan is a derivative of camptothecin, found in Camptotheca acuminata, a plant native to China. It potently inhibits topoisomerase I, an enzyme that facilitates the uncoiling and recoiling of DNA during replication by cleaving one strand and subsequently reattaching that strand. Oxaliplatin is a platinum chemotherapy that inhibits DNA replication and transcription by forming inter- and intrastrand DNA adducts/cross-links.

In patients with $\mathrm{mCRC}$, optimal chemotherapy consists of initial administration of a fluoropyrimidine and oxaliplatin or irinotecan (e.g., FOLFOX or FOLFIRI). Tournigand et al [65] and Colucci et al [66] performed randomized trials where patients received either FOLFIRI followed by FOLFOX, or vice versa. In the Tournigand et al study, FOLIRI was found to have a response rate (RR) of $56 \%$ and a 8.5 -month median progression free survival (mPFS), whereas FOLFOX had a RR of $54 \%$ and a mPFS of 8 months. Colucci et al found that FOLFIRI had a RR of $31 \%$ and FOLFOX had a RR of $34 \%$. Both regimens had a mPFS of 7 months. Both investigators concluded that both regimens had similar efficacy when used as first-line therapy. Therefore, either FOLFOX or FOLFIRI can be considered standard options for first-line treatment of $\mathrm{mCRC}$. These regimens are typically given with bevacizumab.

Bevacizumab is a monoclonal antibody that binds to vascular endothelial growth factor (VEGF) ligand to inhibit angiogenesis. Its antineoplastic effect is ascribed to regression of microvascular density, inhibition of neovascularization, and "normalization" of grossly abnormal tumor vasculature that permits more effective chemotherapy delivery to the tumor. The FDA recently approved bevacizumab in combination with 5FU-based chemotherapy for $\mathrm{mCRC}$ based on findings that addition of bevacizumab to irinotecan, $5 \mathrm{FU}$, and LV for mCRC improved PFS from 6.2 months to 10.6 months, improved the response rate from 35 to 45 [67] and improved overall survival from 15.6 to 20.3 months. Saltz et al found that the addition of bevacizumab to oxaliplatin-based chemotherapy significantly improved PFS from 8.0 to 9.3 months without an improvement in response rate [68]. The finding of improved PFS without improved RR is common in trials of targeted therapy because the metastatic lesions can cavitate or has necrosis rather than regress. Recently, XELOX chemotherapy with or without bevacuzimab was found to be noninferior to FOLFOX with or without bevacuzimab [69]. XELOX chemotherapy includes a combination of oral 5FU known as capecitabine or xeloda (XEL) plus oxaliplatin (OX). XELOX can be used as an alternative in patients who cannot tolerate FOLFOX side effects.

In 2004, the FDA approved cetuximab, the chimeric (human/mouse) monoclonal antibody targeting epidermal growth factor receptor (EGFR), for treatment of mCRC with irinotecan, and as a single agent for patients intolerant of irinotecan-based therapy. Early randomized trials showed benefit of cetuximab in previously treated mCRC patients. When cetuximab was combined with irinotecan in patients refractory to irinotecan-based chemotherapy, the response rate was $22.9 \%$ versus $10.8 \%$ for irinotecan alone [70]. Among patients who failed previous lines of treatment, monotherapy with cetuximab was found to improve overall survival, PFS, and quality of life compared with best support care alone [71]. Cetuximab causes an acneform rash on the face and upper body in more than $80 \%$ of patients. The rash is associated with improved survival. 
Although the FDA approved cetuximab for use in epidermal growth factor receptor (EGFR) expressing $\mathrm{mCRC}$, there is no evidence that the presence or absence of EGFR expression influences RR, and routine testing for this is unnecessary. K-ras mutations have been shown to predict response to cetuximab. The K-ras gene encodes a GTPase protein that is involved in cell signal transduction pathways [72]. Wild-type (nonmutated) K-ras is found in normal cells. Approximately $40 \%$ of colorectal tumor cells have a mutated K-ras gene resulting in constitutively active protein and abnormal cell growth, proliferation, and differentiation. Evidence suggests there is no benefit in using cetuximab monotherapy in previously treated and untreated mCRC patients who have mutated K-ras tumors. Previously treated metastatic colorectal patients with mutated K-ras tumors did not benefit from cetuximab monotherapy, in contrast to patients with wild-type K-ras who had significantly improved overall survival and PFS [74]. FOLIFIRI and cetuximab as first-line therapy in $\mathrm{mCRC}$ was found to reduce the risk of disease progression; however, the benefit was limited to patients with K-ras wild-type tumors (HR 0.68, CI 0.50-0.94) [73].

In 2006, the FDA approved panitumumab, a monoclonal antibody to EGFR, which unlike cetuximab, is fully humanized (not chimeric). It is indicated for patients with $\mathrm{mCRC}$ who have progressed on or are following $5 \mathrm{FU}$, oxaliplatin, and irinotecan-containing regimens. In a large randomized trial of panitumumab versus best supportive care for $\mathrm{mCRC}$, a response rate of $10 \%$ was found [74]. Like cetuximab, panitumumab causes an acneform skin rash. As a fully human monoclonal antibody, panitumumab has a lower risk of serious infusion reactions than the $3 \%$ rate observed with cetuximab. Similar to cetuximab, panitumumab monotherapy is more efficacious in patients with wild- rather than mutant-type K-ras tumors. In a randomized clinical trial of previously treated mCRC patients, median PFS and OS was significantly improved in the wild-type K-ras group compared with the mutant group [75]. 17\% of patients with wild-type $\mathrm{K}$-ras responded to treatment versus $0 \%$ of patients with mutant K-ras. The relative activity of cetuximab versus panitumumab, as well as the relative activity of panitumumab when given with chemotherapy, is currently unknown.

\section{Rectal cancer}

Given the higher local recurrence rates and poorer overall survival of patients with rectal cancer, multimodality management is important. In the early 1990s, the standard of care following surgical resection for full thickness (T3-4) or lymph node positive rectal cancer was postoperative chemoradiotherapy as it was found to improve both local control and OS compared with surgery alone $[77,78]$. Recently, preoperative chemoradiotherapy has become the treatment of choice for full thickness rectal cancers prior to total mesorectal excision based on a randomized clinical trial conducted by Sauer et al. Although this trial showed no difference in OS, improved local recurrence rates (6\% versus $13 \%)$ were found for patients receiving preoperative 5FU-based chemoradiotherapy as compared with postoperative chemoradiotherapy [79]. Preoperative 5FU chemoradiotherapy as compared with preoperative radiation alone also has been shown to improve local recurrence rates (2.7\% versus $14.6 \%)$ [80]. 


\section{Liver metastases}

The standard of care for patients with resectable liver metastases as their only site of cancer spread is changing from previous surgical resection alone to a combination of perioperative chemotherapy and surgery based on a trial conducted by Nordlinger et al [76]. This trial randomized patients with one to four potentially resectable liver metastases to either perioperative chemotherapy (six cycles of FOLFOX chemotherapy both pre- and postresection) or surgery alone. The authors concluded that perioperative chemotherapy reduced the risk of events such as progressive or recurrent disease and death by $25 \%$ in eligible and resected patients without increased severe, life-threatening toxicity. The results of this trial are controversial because when all randomized patients were included in the analysis only a trend and not significance in PFS favoring the chemotherapy arm was found.

\section{Targeted therapy}

Although new drug development takes years, targeted drug use can occur more quickly with advanced tests and will be a focus of future work. In addition, efforts will focus on identifying biomarkers that predict response to systemic therapy so that tailored therapy can be initiated.

With regards to the future of adjuvant systemic chemotherapy, microsatellite-instability (MSI) testing of tumor DNA may be used to identify which patients will benefit from additional therapy (i.e., predictive biomarker) [81, 82]. Approximately $15 \%$ of colon cancers exhibit MSI commonly caused by loss of DNA mismatch-repair pathways. Tumors display short repeated nucleotide sequences called microsatellites secondary to frame-shift mutations and base-pair substitutions. Recent retrospective evidence demonstrated that adjuvant 5FU-based chemotherapy improved OS among patients with microsatellite-stable tumors. However, there was no benefit to those patients with high MSI [83-90]. Ongoing trials are attempting to replicate these findings in a prospective manner. The clinical benefit of cetuximab, a monoclonal antibody against EGFR, varies greatly depending on tumor biology: the greatest benefit is among patients with wild-type (nonmutated) K-ras tumors. In the metastatic setting, potential predictive biomarkers of interest include K-ras, epiregulin, B-raf, PTEN, and Pi3K. Jonker et al found that mCRC patients with both high epiregulin (ligand for EGFR) gene expression and K-ras wild-type status had greater benefit from cetuximab therapy (HR for overall survival 0.43, $p=0.001$ ) [94]. In addition, loss of the tumor-suppressor gene PTEN [95] and having mutated protein kinase B-raf may [96]predict for resistance to EGFR therapy such as cetuximab.

\section{Discussion}

Over the past decade, the prevention and treatment of colorectal cancer has rapidly evolved. To implement evidence-based care a multidisciplinary team is required including surgeons, 
radiation and medical oncologists, as well as gastroenterologists, radiologists, pathologists, and primary care physicians. Unfortunately, despite improvements in surgical techniques and systemic therapy CRC still remains the number two cause of cancer mortality in North America. This study evaluated the usefulness of oncology assess the standardization of CRC chemotherapy and the results at the rate of recurrence and survival. The methodology has enabled the understanding of patterns used for CRC chemotherapy around the world. The results showed significant differences in patterns between countries, regions and institutions. In addition, the actual use of CRC chemotherapy may depend on the health policies of the respective governments. Schemes used are in line with the recommendations of the new guidelines, with the exception of hospital characteristics depended specialization. In first-line chemotherapy for stage IV CRC, general hospitals still favored the use of oral fluoropyrimidines, such as UFT / LV and S-1. However, the differences between general hospitals, cancer centers and university hospitals has decreased after the revision of the guidelines. In adjuvant chemotherapy for stage III CRC, cancer centers and general hospitals used similar patterns, but those that are used in different hospitals. Measures and indicators are greatly needed to evaluate and improve the quality of cancer treatment. Using market research to develop indicators for the standardization of care against cancer is a new methodology. Data not only showed evidence practice gap, but also the growing standardization of CRC affected by chemotherapy treatment guidelines. Methodology indicates a lack of standardization in the care of CRC. Oncology market research also has the potential for cost-effectiveness analyzes, such as sales data for each agent can be evaluated using the analysis system of oncology. Efforts to improve screening utilization by the general population are required to improve mortality and morbidity from CRC. Research advances in medical oncology will result in better understanding of tumor genetics and biology of the host. This will allow systemic therapy to be tailored to specific tumor molecular targets, while sparing toxicity to normal tissue. With these improvements in CRC care, the disease will be treatable with tailored medical treatments that are effective with low toxicity.

\section{Recommendations}

\subsection{Stage II colorectal cancer}

- The routine use of adjuvant chemotherapy for all patients with stage II colon cancer is not recommended. However, the subset of patients with high-risk stage II disease who should be considered for adjuvant therapy includes patients with inadequately sampled nodes, T4 lesions, perforation, or poorly differentiated histology.

- The ultimate clinical decision should be based on discussions with the patient about the nature of the evidence supporting treatment, the anticipated morbidity of treatment, the presence of high-risk prognostic features on individual prognosis, and patient preferences.

- When treated with adjuvant therapy, high-risk stage II patients should receive similar regimens to those recommended for stage III patients. The enrolment of resected high-risk 
stage II patients in clinical trials is encouraged. Additional trials comparing adjuvant therapy with observation are needed and are ethically acceptable in stage II colon cancer.

\subsection{Stage III colorectal cancer}

It could be recommended that patients with completely resected stage III colon cancer should be offered adjuvant chemotherapy and that this treatment should start within eight weeks of surgery. Treatment should depend on factors such as patient suitability and preference, and patients and clinicians must work together to determine the optimal course of treatment. The recommended treatment option is:

- 5-FU given intravenously in combination with leucovorin (LV) and oxaliplatin in the regimens known as FOLFOX or FLOX. These 5-FU/LV/oxaliplatin regimens have demonstrated superior DFS when compared with 5-FU plus LV and are the recommended regimens. Oxaliplatin administration is associated with a $1 \%$ risk of persistent grade 3 neuropathy that needs to be considered in conjunction with expected benefits of therapy.

- Some patients would not be considered appropriate for oxaliplatin regimens. Examples include patients with underlying neurologic conditions or at increased risk of neuropathy, patients at increased risk for infections, and patients likely to poorly tolerate infections as a result of chemotherapy. For these patients, the treatment options are:

- Oral capecitabine administered for six months, which has equivalent efficacy to intravenous 5-FU/LV. Capecitabine results in significantly less diarrhea, stomatitis, neutropenia, nausea/ vomiting, and alopecia but significantly more hand-foot syndrome when compared with 5FU/LV.

- 5-FU in combination with LV administered for six months using either the weekly or monthly schedule.

Suitable patients should be offered entry into clinical trials testing new adjuvant treatments for resected stage III colon cancer.

\section{Author details}

Krassimir Ivanov ${ }^{1}$, Nikola Kolev ${ }^{1}$, Ivan Shterev ${ }^{2}$, Anton Tonev ${ }^{1}$, Valentin Ignatov ${ }^{1}$, Vasil Bojkov ${ }^{4}$ and Tanya Kirilova ${ }^{3}$

1 Department of General and Operative Surgery, University Hospital "St. Marina”, Varna, Bulgaria

2 Department of Medical Oncology, University Hospital “St. Marina”, Varna, Bulgaria

3 Department of Gastroenterology, University Hospital “St. Marina”, Varna, Bulgaria

4 Department of Surgery, University Hospital “St. Marina”, Varna, Bulgaria 


\section{References}

[1] Parkin DM, Bray F, Ferlay J, Pisani P. Global cancer statistics, 2002. CA: a cancer journal for clinicians 2005;55(2):74-108.

[2] Ries LA, Wingo PA, Miller DS, Howe HL, Weir HK, Rosenberg HM, et al. The annual report to the nation on the status of cancer, 1973-1997, with a special section on colorectal cancer. Cancer 2000;88(10):2398-424.

[3] Siegel R, Ward E, Brawley O, Jemal A. Cancer statistics, 2011: the impact of eliminating socioeconomic and racial disparities on premature cancer deaths. CA: a cancer journal for clinicians 2011;61(4):212-36.

[4] Meyerhardt JA, Mayer RJ. Systemic therapy for colorectal cancer. The New England journal of medicine 2005;352(5):476-87.

[5] Biagi JJ, Raphael MJ, Mackillop WJ, Kong W, King WD, Booth CM. Association between time to initiation of adjuvant chemotherapy and survival in colorectal cancer: a systematic review and meta-analysis. JAMA : the journal of the American Medical Association 2011;305(22):2335-42.

[6] Goldberg RM, Sargent DJ, Morton RF, Fuchs CS, Ramanathan RK, Williamson SK, et al. A randomized controlled trial of fluorouracil plus leucovorin, irinotecan, and oxaliplatin combinations in patients with previously untreated metastatic colorectal cancer. Journal of clinical oncology : official journal of the American Society of Clinical Oncology 2004;22(1):23-30.

[7] Andre T, Afchain P, Barrier A, Blanchard P, Larsen AK, Tournigand C, et al. Current status of adjuvant therapy for colon cancer. Gastrointestinal cancer research : GCR 2007;1(3):90-7.

[8] Benson AB, 3rd, Schrag D, Somerfield MR, Cohen AM, Figueredo AT, Flynn PJ, et al. American Society of Clinical Oncology recommendations on adjuvant chemotherapy for stage II colon cancer. Journal of clinical oncology : official journal of the American Society of Clinical Oncology 2004;22(16):3408-19.

[9] Chun P, Wainberg ZA. Adjuvant Chemotherapy for Stage II Colon Cancer: The Role of Molecular Markers in Choosing Therapy. Gastrointestinal cancer research : GCR 2009;3(5):191-6.

[10] Ribic CM, Sargent DJ, Moore MJ, Thibodeau SN, French AJ, Goldberg RM, et al. Tumor microsatellite-instability status as a predictor of benefit from fluorouracil-based adjuvant chemotherapy for colon cancer. The New England journal of medicine 2003;349(3):247-57.

[11] Lynch HT, de la Chapelle A. Hereditary colorectal cancer. The New England journal of medicine 2003;348(10):919-32. 
[12] Halvarsson B, Anderson H, Domanska K, Lindmark G, Nilbert M. Clinicopathologic factors identify sporadic mismatch repair-defective colon cancers. American journal of clinical pathology 2008;129(2):238-44.

[13] Cunningham JM, Christensen ER, Tester DJ, Kim CY, Roche PC, Burgart LJ, et al. Hypermethylation of the hMLH1 promoter in colon cancer with microsatellite instability. Cancer research 1998;58(15):3455-60.

[14] Roth AD, Tejpar S, Delorenzi M, Yan P, Fiocca R, Klingbiel D, et al. Prognostic role of KRAS and BRAF in stage II and III resected colon cancer: results of the translational study on the PETACC-3, EORTC 40993, SAKK 60-00 trial. Journal of clinical oncology : official journal of the American Society of Clinical Oncology 2010;28(3):466-74.

[15] Moertel CG, Fleming TR, Macdonald JS, Haller DG, Laurie JA, Goodman PJ, et al. Levamisole and fluorouracil for adjuvant therapy of resected colon carcinoma. The New England journal of medicine 1990;322(6):352-8.

[16] Moertel CG, Fleming TR, Macdonald JS, Haller DG, Laurie JA, Tangen CM, et al. Fluorouracil plus levamisole as effective adjuvant therapy after resection of stage III colon carcinoma: a final report. Annals of internal medicine 1995;122(5):321-6.

[17] Ackland SP, Beale P, Peters GJ. Thymidylate synthase inhibitors. Cancer chemotherapy and biological response modifiers 2003;21:1-28.

[18] Jager E, Heike M, Bernhard H, Klein O, Bernhard G, Lautz D, et al. Weekly high-dose leucovorin versus low-dose leucovorin combined with fluorouracil in advanced colorectal cancer: results of a randomized multicenter trial. Study Group for Palliative Treatment of Metastatic Colorectal Cancer Study Protocol 1. Journal of clinical oncology : official journal of the American Society of Clinical Oncology 1996;14(8):2274-9.

[19] Andre T, Colin P, Louvet C, Gamelin E, Bouche O, Achille E, et al. Semimonthly versus monthly regimen of fluorouracil and leucovorin administered for 24 or 36 weeks as adjuvant therapy in stage II and III colon cancer: results of a randomized trial. Journal of clinical oncology : official journal of the American Society of Clinical Oncology 2003;21(15):2896-903.

[20] Saini A, Norman AR, Cunningham D, Chau I, Hill M, Tait D, et al. Twelve weeks of protracted venous infusion of fluorouracil (5-FU) is as effective as 6 months of bolus 5 -FU and folinic acid as adjuvant treatment in colorectal cancer. British journal of cancer 2003;88(12):1859-65.

[21] Efficacy of adjuvant fluorouracil and folinic acid in B2 colon cancer. International Multicentre Pooled Analysis of B2 Colon Cancer Trials (IMPACT B2) Investigators. Journal of clinical oncology : official journal of the American Society of Clinical Oncology 1999;17(5):1356-63. 
[22] Efficacy of adjuvant fluorouracil and folinic acid in colon cancer. International Multicentre Pooled Analysis of Colon Cancer Trials (IMPACT) investigators. Lancet 1995;345(8955):939-44.

[23] Tournigand C, Andre T, Bonnetain F, Chibaudel B, Lledo G, Hickish T, et al. Adjuvant therapy with fluorouracil and oxaliplatin in stage II and elderly patients (between ages 70 and 75 years) with colon cancer: subgroup analyses of the Multicenter International Study of Oxaliplatin, Fluorouracil, and Leucovorin in the Adjuvant Treatment of Colon Cancer trial. Journal of clinical oncology : official journal of the American Society of Clinical Oncology 2012;30(27):3353-60.

[24] Yothers G, O'Connell MJ, Allegra CJ, Kuebler JP, Colangelo LH, Petrelli NJ, et al. Oxaliplatin as adjuvant therapy for colon cancer: updated results of NSABP C-07 trial, including survival and subset analyses. Journal of clinical oncology : official journal of the American Society of Clinical Oncology 2011;29(28):3768-74.

[25] Hoff PM, Ansari R, Batist G, Cox J, Kocha W, Kuperminc M, et al. Comparison of oral capecitabine versus intravenous fluorouracil plus leucovorin as first-line treatment in 605 patients with metastatic colorectal cancer: results of a randomized phase III study. Journal of clinical oncology : official journal of the American Society of Clinical Oncology 2001;19(8):2282-92.

[26] Douillard JY, Hoff PM, Skillings JR, Eisenberg P, Davidson N, Harper P, et al. Multicenter phase III study of uracil/tegafur and oral leucovorin versus fluorouracil and leucovorin in patients with previously untreated metastatic colorectal cancer. Journal of clinical oncology : official journal of the American Society of Clinical Oncology 2002;20(17):3605-16.

[27] Van Cutsem E, Twelves C, Cassidy J, Allman D, Bajetta E, Boyer M, et al. Oral capecitabine compared with intravenous fluorouracil plus leucovorin in patients with metastatic colorectal cancer: results of a large phase III study. Journal of clinical oncology : official journal of the American Society of Clinical Oncology 2001;19(21):4097-106.

[28] Pasetto LM, Monfardini S. The role of capecitabine in the treatment of colorectal cancer in the elderly. Anticancer research 2006;26(3B):2381-6.

[29] Reigner B, Blesch K, Weidekamm E. Clinical pharmacokinetics of capecitabine. Clinical pharmacokinetics 2001;40(2):85-104.

[30] Twelves C, Scheithauer W, McKendrick J, Seitz JF, Van Hazel G, Wong A, et al. Capecitabine versus 5-fluorouracil/folinic acid as adjuvant therapy for stage III colon cancer: final results from the X-ACT trial with analysis by age and preliminary evidence of a pharmacodynamic marker of efficacy. Annals of oncology : official journal of the European Society for Medical Oncology / ESMO 2012;23(5):1190-7.

[31] Poplin EA, Benedetti JK, Estes NC, Haller DG, Mayer RJ, Goldberg RM, et al. Phase III Southwest Oncology Group 9415/Intergroup 0153 randomized trial of fluorouracil, leucovorin, and levamisole versus fluorouracil continuous infusion and levami- 
sole for adjuvant treatment of stage III and high-risk stage II colon cancer. Journal of clinical oncology : official journal of the American Society of Clinical Oncology 2005;23(9): 1819-25.

[32] Lembersky BC, Wieand HS, Petrelli NJ, O'Connell MJ, Colangelo LH, Smith RE, et al. Oral uracil and tegafur plus leucovorin compared with intravenous fluorouracil and leucovorin in stage II and III carcinoma of the colon: results from National Surgical Adjuvant Breast and Bowel Project Protocol C-06. Journal of clinical oncology : official journal of the American Society of Clinical Oncology 2006;24(13):2059-64.

[33] Raymond E, Chaney SG, Taamma A, Cvitkovic E. Oxaliplatin: a review of preclinical and clinical studies. Annals of oncology : official journal of the European Society for Medical Oncology / ESMO 1998;9(10):1053-71.

[34] Culy CR, Clemett D, Wiseman LR. Oxaliplatin. A review of its pharmacological properties and clinical efficacy in metastatic colorectal cancer and its potential in other malignancies. Drugs 2000;60(4):895-924.

[35] Alcindor T, Beauger N. Oxaliplatin: a review in the era of molecularly targeted therapy. Curr Oncol 2011;18(1):18-25.

[36] Brouwers EE, Huitema AD, Beijnen JH, Schellens JH. Long-term platinum retention after treatment with cisplatin and oxaliplatin. BMC clinical pharmacology 2008;8:7.

[37] Andre T, Boni C, Navarro M, Tabernero J, Hickish T, Topham C, et al. Improved overall survival with oxaliplatin, fluorouracil, and leucovorin as adjuvant treatment in stage II or III colon cancer in the MOSAIC trial. Journal of clinical oncology : official journal of the American Society of Clinical Oncology 2009;27(19):3109-16.

[38] Adjuvant chemotherapy with oxaliplatin, in combination with fluorouracil plus leucovorin prolongs disease-free survival, but causes more adverse events in people with stage II or III colon cancer Abstracted from: Andre T, Boni C, Mounedji-Boudiaf $\mathrm{L}$, et al. Multicenter international study of oxaliplatin/5-fluorouracil/leucovorin in the adjuvant treatment of colon cancer (MOSAIC) investigators. Oxaliplatin, fluorouracil, and leucovorin as adjuvant treatment for colon cancer. $\mathrm{N}$ Engl J Med 2004;350:2343-51. Cancer treatment reviews 2004;30(8):711-3.

[39] Kuebler JP, Wieand HS, O'Connell MJ, Smith RE, Colangelo LH, Yothers G, et al. Oxaliplatin combined with weekly bolus fluorouracil and leucovorin as surgical adjuvant chemotherapy for stage II and III colon cancer: results from NSABP C-07. Journal of clinical oncology : official journal of the American Society of Clinical Oncology 2007;25(16):2198-204.

[40] Schmoll HJ, Cartwright T, Tabernero J, Nowacki MP, Figer A, Maroun J, et al. Phase III trial of capecitabine plus oxaliplatin as adjuvant therapy for stage III colon cancer: a planned safety analysis in 1,864 patients. Journal of clinical oncology : official journal of the American Society of Clinical Oncology 2007;25(1):102-9. 
[41] Haller DG, Tabernero J, Maroun J, de Braud F, Price T, Van Cutsem E, et al. Capecitabine plus oxaliplatin compared with fluorouracil and folinic acid as adjuvant therapy for stage III colon cancer. Journal of clinical oncology : official journal of the American Society of Clinical Oncology 2011;29(11):1465-71.

[42] Xu Y, Villalona-Calero MA. Irinotecan: mechanisms of tumor resistance and novel strategies for modulating its activity. Annals of oncology : official journal of the European Society for Medical Oncology / ESMO 2002;13(12):1841-51.

[43] Saltz LB, Niedzwiecki D, Hollis D, Goldberg RM, Hantel A, Thomas JP, et al. Irinotecan fluorouracil plus leucovorin is not superior to fluorouracil plus leucovorin alone as adjuvant treatment for stage III colon cancer: results of CALGB 89803. Journal of clinical oncology : official journal of the American Society of Clinical Oncology 2007;25(23): 3456-61.

[44] Van Cutsem E, Labianca R, Bodoky G, Barone C, Aranda E, Nordlinger B, et al. Randomized phase III trial comparing biweekly infusional fluorouracil/leucovorin alone or with irinotecan in the adjuvant treatment of stage III colon cancer: PETACC-3. Journal of clinical oncology : official journal of the American Society of Clinical Oncology 2009;27(19):3117-25.

[45] McCormack PL, Keam SJ. Bevacizumab: a review of its use in metastatic colorectal cancer. Drugs 2008;68(4):487-506.

[46] Allegra CJ, Yothers G, O'Connell MJ, Sharif S, Petrelli NJ, Colangelo LH, et al. Phase III trial assessing bevacizumab in stages II and III carcinoma of the colon: results of NSABP protocol C-08. Journal of clinical oncology : official journal of the American Society of Clinical Oncology 2011;29(1):11-6.

[47] Merlano M, Occelli M. Review of cetuximab in the treatment of squamous cell carcinoma of the head and neck. Therapeutics and clinical risk management 2007;3(5):871-6.

[48] Alberts SR, Sargent DJ, Nair S, Mahoney MR, Mooney M, Thibodeau SN, et al. Effect of oxaliplatin, fluorouracil, and leucovorin with or without cetuximab on survival among patients with resected stage III colon cancer: a randomized trial. JAMA : the journal of the American Medical Association 2012;307(13):1383-93.

[49] Ades S. Adjuvant chemotherapy for colon cancer in the elderly: moving from evidence to practice. Oncology (Williston Park) 2009;23(2):162-7.

[50] Gill S, Loprinzi CL, Sargent DJ, Thome SD, Alberts SR, Haller DG, et al. Pooled analysis of fluorouracil-based adjuvant therapy for stage II and III colon cancer: who benefits and by how much? Journal of clinical oncology : official journal of the American Society of Clinical Oncology 2004;22(10):1797-806.

[51] Kahn KL, Adams JL, Weeks JC, Chrischilles EA, Schrag D, Ayanian JZ, et al. Adjuvant chemotherapy use and adverse events among older patients with stage III colon cancer. JAMA : the journal of the American Medical Association 2010;303(11):1037-45. 
[52] Dobie SA, Baldwin LM, Dominitz JA, Matthews B, Billingsley K, Barlow W. Completion of therapy by Medicare patients with stage III colon cancer. Journal of the National Cancer Institute 2006;98(9):610-9.

[53] Abraham A, Habermann EB, Rothenberger DA, Kwaan M, Weinberg AD, Parsons $\mathrm{HM}$, et al. Adjuvant chemotherapy for stage III colon cancer in the oldest old: Results beyond clinical guidelines. Cancer 2012.

[54] Jemal A, Siegel R, Ward E, et al. Cancer statistics, 2008. CA Cancer J Clin. 2008;58(2): 71-96.

[55] Canadian Cancer Society/National Cancer Institute of Canada. Canadian Cancer Statistics. Toronto, Canada: 2008.

[56] Therasse P, Arbuck S G, Eisenhauer E A, et al. New guidelines to evaluate the response to treatment in solid tumors. European Organization for Research and Treatment of Cancer, National Cancer Institute of the United States, National Cancer Institute of Canada. J Natl Cancer Inst. 2000;92(3):205-216.

[57] Wolmark N, Fisher B, Rockette H, et al. Postoperative adjuvant chemotherapy or BCG for colon cancer: results from NSABP protocol C-01. J Natl Cancer Inst. 1988;80(1):30-36.

[58] Wolmark N, Rockette H, Fisher B, et al. The benefit of leucovorin-modulated fluorouracil as postoperative adjuvant therapy for primary colon cancer: results from $\mathrm{Na}-$ tional Surgical Adjuvant Breast and Bowel Project protocol C-03. J Clin Oncol. 1993;11(10):1879-1887.

[59] Scheithauer W, Rosen H, Kornek G V, Sebesta C, Depisch D. Randomised comparison of combination chemotherapy plus supportive care with supportive care alone in patients with metastatic colorectal cancer.BMJ. 1993;306(6880):752-755.

[60] Jonker D S, Maroun K J. Adjuvant Systemic Chemotherapy for Stage II and III Colon Cancer Following Complete Resection: Guideline Recommendations. Toronto: Cancer Care Ontario Evidence-Based Series\#2-29; Cancer Care Ontario; 2008.

[61] André T, Boni C, Mounedji-Boudiaf L, et al. Multicenter International Study of Oxaliplatin/5-Fluorouracil/Leucovorin in the Adjuvant Treatment of Colon Cancer (MOSAIC) Investigators Oxaliplatin, fluorouracil, and leucovorin as adjuvant treatment for colon cancer. N Engl J Med. 2004;350(23):2343-2351.

[62] De Gramont A, Boni C, Navarro M, et al. Oxaliplatin/5-FU/LV in adjuvant colon cancer: Updated efficacy results of the MOSAIC trial, including survival, with a median follow-up of six years. Journal of Clinical Oncology, 2007 ASCO Annual Meeting Proceedings Part 1. 2007;25(18S) Abstract 4007.

[63] Krueger G M, Alexander L L, Whippen D A, Balch C M. Arnoldus Goudsmit, MD, PhD: chemotherapist, visionary, founder of the American Society of Clinical Oncology, 1909-2005. J Clin Oncol.2006;24(24):4033-4036. 
[64] Tournigand C, André T, Achille E, et al. FOLFIRI followed by FOLFOX6 or the reverse sequence in advanced colorectal cancer: a randomized GERCOR study. J Clin Oncol. 2004;22(2):229-237.

[65] Colucci G, Gebbia V, Paoletti G, et al. Gruppo Oncologico Dell'Italia Meridionale Phase III randomized trial of FOLFIRI versus FOLFOX4 in the treatment of advanced colorectal cancer: a multicenter study of the Gruppo Oncologico Dell'Italia Meridionale. J Clin Oncol. 2005;23(22):4866-4875.

[66] Hurwitz H, Fehrenbacher L, Novotny W, et al. Bevacizumab plus irinotecan, fluorouracil, and leucovorin for metastatic colorectal cancer. N Engl J Med. 2004;350(23): 2335-2342.

[67] Saltz L, Clarke S, Diaz-Rubio E, et al. Bevacizumab (Bev) in combination with XELOX or FOLFOX4: efficacy results from XELOX-1/NO16966, a randomized phase III trial in the first-line treatment of metastatic colorectal cancer (MCRC). Orlando, FL: Paper presented at: Gastrointestinal Cancers Symposium, American Society of Clinical Oncologists; July 19-21, 2007. Abstract 238.

[68] Cassidy J, Clarke S, Díaz-Rubio E, et al. Randomized phase III study of capecitabine plus oxaliplatin compared with fluorouracil/folinic acid plus oxaliplatin as first-line therapy for metastatic colorectal cancer.J Clin Oncol. 2008;26(12):2006-2012.

[69] Cunningham D, Humblet $Y$, Siena S, et al. Cetuximab monotherapy and cetuximab plus irinotecan in irinotecan-refractory metastatic colorectal cancer. $\mathrm{N}$ Engl J Med. 2004;351(4):337-345.

[70] Jonker D J, O'Callaghan C J, Karapetis C S, et al. Cetuximab for the treatment of colorectal cancer. N Engl J Med. 2007;357(20):2040-2048.

[71] Cutsem E Van, Köhne C H, Hitre E, et al. Cetuximab and chemotherapy as initial treatment for metastatic colorectal cancer. N Engl J Med. 2009;360(14):1408-1417

[72] Karapetis C S, Khambata-Ford S, Jonker D J, et al. K-ras mutations and benefit from cetuximab in advanced colorectal cancer. N Engl J Med. 2008;359(17):1757-1765.

[73] Cutsem E Van, Peeters M, Siena S, et al. Open-label phase III trial of panitumumab plus best supportive care compared with best supportive care alone in patients with chemotherapy-refractory metastatic colorectal cancer. J Clin Oncol. 2007;25:16581664.

[74] Amado R G, Wolf M, Peeters M, et al. Wild-type KRAS is required for panitumumab efficacy in patients with metastatic colorectal cancer. J Clin Oncol. 2008;26(10):16261634.

[75] Nordlinger B, Sorbye H, Glimelius B, et al. EORTC Gastro-Intestinal Tract Cancer Group. Cancer Research UK. Arbeitsgruppe Lebermetastasen und-tumoren in der Chirurgischen Arbeitsgemeinschaft Onkologie (ALM-CAO) Australasian Gastro-Intestinal Trials Group (AGITG) Fédération Francophone de Cancérologie Digestive 
(FFCD) Perioperative chemotherapy with FOLFOX4 and surgery versus surgery alone for resectable liver metastases from colorectal cancer (EORTC Intergroup trial 40983): a randomised controlled trial. Lancet. 2008;371(9617):1007-1016.

[76] Gastro-intestinal Tumor Study Group. Prolongation of the disease-free interval in surgically treated rectal carcinoma. N Engl J Med. 1985;312:1465-1472.

[77] Krook J E, Moertel C G, Gunderson L L, et al. Effective surgical adjuvant therapy for high-risk rectal carcinoma. N Engl J Med. 1991;324(11):709-715.

[78] Sauer R, Becker H, Hohenberger W, et al. German Rectal Cancer Study Group Preoperative versus postoperative chemoradiotherapy for rectal cancer. $\mathrm{N}$ Engl J Med. 2004;351(17):1731-1740.

[79] Gérard J P, Conroy T, Bonnetain F, et al. Preoperative radiotherapy with or without concurrent fluorouracil and leucovorin in T3-4 rectal cancers: results of FFCD 9203. J Clin Oncol. 2006;24(28):4620-4625.

[80] Gryfe R, Kim H, Hsieh E T, et al. Tumor microsatellite instability and clinical outcome in young patients with colorectal cancer. N Engl J Med. 2000;342(2):69-77.

[81] Hemminki A, Mecklin J P, Järvinen H, Aaltonen L A, Joensuu H. Microsatellite instability is a favorable prognostic indicator in patients with colorectal cancer receiving chemotherapy. Gastroenterology.2000;119(4):921-928.

[82] Ribic C M, Sargent D J, Moore M J, et al. Tumor microsatellite-instability status as a predictor of benefit from fluorouracil-based adjuvant chemotherapy for colon cancer. N Engl J Med. 2003;349(3):247-257.

[83] Jonker D J, Karpetis C, Harbison C, et al. High epiregulin (EREG) gene expression plus K-ras wild-type (WT) status as predictors of cetuximab benefit in the treatment of advanced colorectal cancer (ACRC): results from NCIC CTG CO.17- A phase III trial of cetuximab versus best supportive care (BSC) J Clin Oncol. 2009;27(15S) Abstract 4016.

[84] Loupakis F, Pollina L, Stasi I, et al. PTEN expression and KRAS mutations on primary tumors and metastases in the prediction of benefit from cetuximab plus irinotecan for patients with metastatic colorectal cancer. J Clin Oncol. 2009;27(16):2622-2629.

[85] Di Nicolantonio F, Martini M, Molinari F, et al. Wild-type BRAF is required for response to panitumumab or cetuximab in metastatic colorectal cancer. J Clin Oncol. 2008;26(35):5705-5712. 